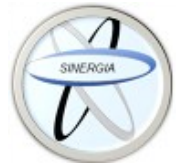

\title{
Síndrome de BRASH BRASH syndrome
}

${ }^{1}$ Dr. Roberto Orozco García Investigador independiente, Alajuela, Costa Rica

(iD) https://orcid.org/0000-0003-4641-5001

${ }^{2}$ Dra. Irene María Acuña Núñez Investigadora independiente, San José, Costa Rica

(D) https://orcid.org/0000-0002-0934-4593

${ }^{3}$ Dr. Sebastián Chacón Álvarez Investigador independiente, San José, Costa Rica

(D) https://orcid.org/0000-0001-8484-2966

Recibido 08/12/2021
Corregido $15 / 01 / 2022$
Aceptado 10/02/2022

\section{RESUMEN}

El síndrome de BRASH surge de un fenómeno sinergista desencadenante de un ciclo vicioso que involucra la interacción entre bradicardia, falla renal, fármacos bloqueadores de la conducción atrioventricular, choque e hiperkalemia. Debido a que es una patología de reciente análisis, su identificación usualmente pasa desapercibida interpretándose como otra afección, lo que da pie a implementar modelos terapéuticos no fundamentados en la fisiopatología subyacente, resultando en el aumento de la morbimortalidad del paciente. El abordaje terapéutico involucra terapia de soporte básico para el manejo de la hiperkalemia, bradicardia y resucitación con fluidoterapia. Usualmente, las medidas básicas son suficientes para romper el ciclo vicioso. Proporcionar una discusión del modelo fisiopatológico mejora el entendimiento y el reconocimiento de esta patología, optimizando el pronóstico de los pacientes que la padezcan.

PALABRAS CLAVE: síndrome de BRASH; hiperpotasemia; bloqueo atrioventricular; pruebas de función renal; intoxicación; antagonistas adrenérgicos beta

\section{ABSTRACT}

BRASH syndrome arises from a synergistic phenomenon that triggers a vicious cycle which involves the interaction between bradycardia, renal failure, atrial ventricular conduction blocking drugs, shock, and hyperkalemia. Due to being a recently described pathology, its identification usually goes unnoticed, interpreting it as another condition, giving rise to the implementation of therapeutic models not based on the underlying pathophysiology resulting in an increase in the 
morbidity and mortality of the patient. The therapeutic approach involves basic supportive therapy for the management of hyperkalemia, bradycardia, and resuscitation with fluid therapy. Basic measures are usually enough to break the vicious cycle. Providing a discussion of the pathophysiological model improves the understanding and recognition of this pathology, optimizing the prognosis of patients who suffer from it.

KEYWORDS: BRASH syndrome; hyperkalemia; atrioventricular block; kidney function tests; poisoning; adrenergic beta-antagonists

${ }^{1}$ Médico general, graduado de la Universidad de Iberoamérica (UNIBE). Cód. MED16746. Correo rob.orozco.garcia@gmail.com

${ }^{2}$ Médica general, graduada de la Universidad de Iberoamérica (UNIBE). Cód. cod. MED16723. Correo: irene.cr@hotmail.com

${ }^{3}$ Médico general, graduado de la Universidad Latina de Costa Rica (U.Latina), Còd. MED16910. Correo: dr.chacon.alvarez@gmail.com

\section{INTRODUCCIÓN}

Síndrome de BRASH es el acrónimo, acuñado desde el 2016 y derivado de las siglas: Bradycardia (bradicardia), Renal Failure (falla renal), Atrioventricular node blockade (bloqueo del nodo atrioventricular), Shock (choque) e Hyperkalemia (hiperkalemia), para describir al conjunto de signos que se originan de los efectos sinergistas entre medicamentos bloqueadores del nodo atrioventricular y la falla renal, resultando en un ciclo vicioso; en el cual, la utilización de medidas terapéuticas ampliamente protocolizadas por entidades internacionales puede llevar a la muerte, si se utilizan de forma dogmática (1).

Es conveniente señalar que lo anterior no quiere decir que el síndrome de BRASH sea una entidad fisiopatológica de reciente aparición, la cual no afectaba previamente a las personas o una entidad que demande aplicar medidas terapéuticas complejas, sino que no había entrado en un marco de definición clínica. Ciertamente, es de sospechar que el síndrome de BRASH ha afectado desde la introducción de fármacos con acción a nivel del sistema cardiovascular y, si bien es cierto, no se había identificado como una entidad propia distinta de otras patologías, la mayoría de los médicos tratantes con experiencia en servicios de emergencias la han abordado de forma exitosa; sin el reconocimiento de esta y utilizando medidas de soporte básicas. Muestra de ello es un reporte de caso descrito en la revista The American Journal of Medicine en 1986 por Lee TH, Salomon DR, Rayment CM y Antman EM, donde describen un caso de hipotensión y arresto sinusal con hiperkalemia inducida por ejercicio en combinación con medicamentos: verapamilo y propranolol (2); probablemente, pasó a la historia como el primer caso reportado de síndrome de $\mathrm{BRASH}$, al haber reflexionado sobre que el origen del evento yacía en una interacción metabólico-medicamentosa.

En la actualidad, existe escasa literatura científica respecto al síndrome de BRASH y la mayoría procede de reportes de casos; lo cual hace que sea considerablemente desconocido en el gremio médico y, por este motivo, subdiagnosticado. Si se toma en cuenta que el síndrome de BRASH es desencadenado por interacciones fármaco metabólicas y que, en la realidad actual, el tamaño de la población adulta mayor y el número de pacientes jóvenes con condiciones de salud complejas viene en incremento de la mano de la polifarmacia (3), la identificación y manejo del síndrome de BRASH adquiere un valor indispensable para mejorar la sobrevida de aquellos que lo 
padezcan (4). El objetivo de esta revisión, por lo tanto, es presentar de forma práctica el Síndrome de BRASH, enfocándose en los mecanismos fisiopatológicos desencadenantes que suscitan en este síndrome, con el propósito de entender e identificar el cuadro clínico, así ayudando a su diagnóstico.

\section{MÉTODO}

Para el desarrollo del presente artículo, se hizo uso de fuentes bibliográficas obtenidas en bases de datos como Pubmed, Google Scholar, Medline (NLM) y se seleccionaron publicaciones a partir de los 5 años más recientes, basado en los términos: "BRASH Syndrome", "Bradycardia", "AV Blockade", "Hyperkalmia" "SHOCK", y "Kidney injury". Posterior a esto, se agregaron términos a la búsqueda para mejorar especificidad: "síntomas", "causas", "diagnóstico" y "tratamiento". De los resultados obtenidos, se excluyen aquellos artículos no relevantes a la revisión y los que se presentan duplicados, en total se obtienen 19 artículos en idioma inglés, de los cuales, es importante mencionar que 15 de ellos son reporte de casos.

\section{EPIDEMILOGÍA}

Poco se sabe sobre la epidemiología verdadera del síndrome de BRASH; esto se debe a que es una entidad clínica recientemente identificada, lo que resulta en pobre exposición y subreporte.

En la actualidad, los datos manejados provienen de reportes de casos, los cuales apuntan a que hay una alta prevalencia en la población adulta mayor que cursa con patología cardíaca y renal subyacente $(4,5)$. Aquellos pacientes que se encuentran en mayor riesgo de desarrollar el síndrome son quienes utilizan múltiples fármacos con acción a nivel de bloqueo de conducción del nodo atrioventricular y utilizan medicamentos antihipertensivos de las categorías antagonistas del receptor de angiotensina II e inhibidores de la enzima convertidora de angiotensina (1).

\section{FISIOPATOLOGÍA}

La fisiopatología aún no está completamente dilucidada, sin embargo, el mecanismo responsable de la espiral de eventos resulta de un efecto sinergista entre medicamentos con efectos que enlentecen la conducción a través del nodo atrioventricular y la hiperkalemia. La hiperkalemia tiene un efecto fisiológico que se ve reflejado en un decremento de la tasa de Fase 0 del potencial de acción cardíaco, llevando a un potencial de acción más prolongado, un complejo QRS más ancho, y a un intervalo PR más prolongado, potenciando el efecto de los medicamentos bloqueadores de la conducción del nodo atrioventricular (6). Lo anterior se manifiesta clínicamente como una profunda bradicardia y con ello un proceso cíclico, en el cual la bradicardia disminuye directamente el gasto cardíaco, por consiguiente, ocasiona un declive en la perfusión renal efectiva resultando en falla renal, empeorando así la hiperkalemia, a su vez, lleva a la acumulación plasmática de medicamentos que actúan sobre la conducción del nodo atrioventricular perpetuando lo que se describe como el ciclo vicioso del síndrome de BRASH $(1,2,6,7)$. El ciclo de eventos, generalmente, se ha visto que es iniciado por alguna circunstancia clínica leve, un factor desencadenante, entre ellos los más reportados en la literatura son: hipovolemia, ya sea por disminución en la ingesta de líquidos o por aumento en las pérdidas de volumen intravascular, como ha ocurrido en caso de pacientes con enfermedad diarreica o por deshidratación 
durante meses de verano, sepsis, incluida en esta categoría la infección por el virus SARS COV-2 causante de COVID-19, aumento en la potencia de medicamentos, ya sea en uso o la introducción de un nuevo medicamento, eventos nefrotóxicos, o en su defecto, cualquier causante de lesión renal aguda. $(1,2,7,8)$. De los casos reportados en los últimos 5 años, las categorías de medicamentos más comúnmente utilizados por pacientes que desarrollan síndrome de BRASH se presentan en la tabla 1.

En la tabla 2, se describen los reportes de casos que se ajustan a la definición de síndrome de BRASH utilizados en esta revisión.

El síndrome de BRASH no es una entidad que surja por alteraciones fisiológicas aisladas, es un proceso sinergista, como se ha dicho previamente; esto quiere decir que, aunque parte esencial del síndrome es la hiperkalemia y el bloqueo atrioventricular, estas por sí solas no logran explicar el desencadenamiento de sucesos fisiopatológicos que se hacen manifiestos.

Actualmente, la forma en la que se interpreta el síndrome de BRASH es: "un continuum que abarca desde la hiperkalemia aislada a la sobredosis de medicamentos bloqueadores del Nodo Atrio ventricular, donde no siempre es posible determinar donde se encuentran los límites" (1). Por ejemplo, en la hiperkalemia pura, como alteración única, los niveles de potasio que se requieren alcanzar para producir bradicardia y cambios electrocardiográficos son más elevados comparados con los que se necesitan en un síndrome de BRASH, 6.0 a $6.5 \mathrm{mEq} / \mathrm{L}$ para identificar aparición de ondas $\mathrm{T}$ picudas en el electrocardiograma, y más de $8 \mathrm{mEq} / \mathrm{L}$ es cuando empiezan a darse las bradicardias sinusales severas (9). Mientras que, en el síndrome de BRASH, se ha reportado que niveles de potasio de $5 \mathrm{mEq} / \mathrm{L}$ ya pueden desencadenar bradiarritmias potencialmente letales (1).

Tabla 1. Medicamentos implicados en síndrome de BRASH

\begin{tabular}{|l|l|}
\hline \multicolumn{1}{|c|}{ FARMACOS } & \multicolumn{1}{|c|}{ TIPOS } \\
\hline Beta bloqueadores & $\begin{array}{l}\text { Propranolol, Metoprolol, Atenolol, } \\
\text { Carvedilol, Sotalol, Bisoprolol. }\end{array}$ \\
\hline $\begin{array}{l}\text { Bloqueadores de canales de calcio (no } \\
\text { dihidropiridinas) }\end{array}$ & Verapamil, Diltiazem \\
\hline Antagonistas del receptor de angiotensina II & Irbersartan, Valsartan, Losartan \\
\hline $\begin{array}{l}\text { Inhibidores de neprilisina y receptor de } \\
\text { angiotensina }\end{array}$ & Sacubitrilo/Valsartan \\
\hline $\begin{array}{l}\text { Inhibidores de la enzima convertidora de } \\
\text { angiotensina }\end{array}$ & Captopril, Lisinopril, Enalapril, Ramipril \\
\hline Antagonistas de receptores de aldosterona & Espironolactona, Eplerona \\
\hline Sulfonamidas y Diaminopirimidinas & Trimetoprima y Sulfametoxazol \\
\hline $\begin{array}{l}\text { Inhibidores del cotransportador Sodio-Glucosa } \\
\text { tipo } 2\end{array}$ & Ipragliflozina \\
\hline
\end{tabular}


Fuente: Elaborado en base a Farkas JD, Long B, Koyfman A, Menson K. BRASH Syndrome: Bradycardia, Renal Failure, AV Blockade, Shock, and Hyperkalemia. J Emerg Med. 2020 Aug;59(2):216-23.

\begin{tabular}{|c|c|c|c|c|c|c|}
\hline Edad & Medicamentos & $\begin{array}{l}\text { Potasio } \\
(\mathrm{mEq} / \mathrm{L})\end{array}$ & $\begin{array}{c}\mathrm{Cr} \\
(\mathrm{mg} / \mathrm{dl}) / \\
\text { eGFR }\end{array}$ & $\begin{array}{l}\text { Signos } \\
\text { vitales }\end{array}$ & Tratamiento & Referencia \\
\hline 62 & $\begin{array}{l}\text { Metolazone, } \\
\text { Bumetanide, } \\
\text { Carvedilol }\end{array}$ & 8.0 & 4.06 & $\begin{array}{l}\text { FC: } 31 \\
\text { PA:63/32 }\end{array}$ & $\begin{array}{l}\text { Insulina, sulfonato } \\
\text { de poliestireno } \\
\text { sódico, gluconato de } \\
\text { calcio, y dopamina }\end{array}$ & $\begin{array}{l}\text { Srivastava } \\
\text { et al. } 2020 \\
(7)\end{array}$ \\
\hline 43 & $\begin{array}{l}\text { Diltiazem, } \\
\text { Metoprolol }\end{array}$ & 7.6 & 2.75 & $\begin{array}{l}\text { FC:35 } \\
\text { PA:109/42 }\end{array}$ & $\begin{array}{l}\text { Norepinefrina, } \\
\text { insulina, dextrosa, } \\
\text { bicarbonato, } \\
\text { gluconato de calcio, } \\
\text { marcapaso } \\
\text { transcutáneo, y } \\
\text { TRR. }\end{array}$ & $\begin{array}{l}\text { Grigorov et } \\
\text { al. } 2020 \text { (9) }\end{array}$ \\
\hline 64 & $\begin{array}{l}\text { Bisoprolol, } \\
\text { Sacubitril/valsart } \\
\text { an, furosemida, } \\
\text { espironolactona }\end{array}$ & 5.8 & 7.07 & $\begin{array}{l}\text { FC: } 28 \\
\text { PA: } 72 / 33\end{array}$ & $\begin{array}{l}\text { Bicarbonato, } \\
\text { gluconato de calcio, } \\
\text { insulina + dextrosa, } \\
\text { nebulización con } \\
\text { salbutamol, infusión } \\
\text { de fenilefrina, TRR, } \\
\text { marcapasos } \\
\text { transcutáneo, } \\
\text { norepinefrina, } \\
\text { dobutamina, } \\
\text { vasopresina. }\end{array}$ & $\begin{array}{l}\text { Ata et al. } \\
2021(12)\end{array}$ \\
\hline 52 & $\begin{array}{l}\text { Nifedipina, } \\
\text { Furosemida, } \\
\text { Labetalol, } \\
\text { Metoprolol se } \\
\text { cambió por } \\
\text { Carvedilol }\end{array}$ & 5.3 & 2.2 & $\begin{array}{l}\text { FC:44 } \\
\text { PA: } 67 / 49\end{array}$ & $\begin{array}{l}\text { Epinefrina, cloruro } \\
\text { de calcio, } \\
\text { marcapasos } \\
\text { transvenoso, } \\
\text { dopamina, } \\
\text { norepinefrina, } \\
\text { glucagón. }\end{array}$ & $\begin{array}{l}\text { Sarkar et al. } \\
2021 \text { (6) }\end{array}$ \\
\hline 81 & $\begin{array}{l}\text { Atenolol, } \\
\text { Ramipril, AINEs }\end{array}$ & 8.3 & $12 \mathrm{ml} / \mathrm{min}$ & $\begin{array}{l}\text { FC: } 29 \\
\text { PA: } 100 / 60 \\
\text { (post } \\
\text { Atropina) }\end{array}$ & No se describe & $\begin{array}{l}\text { Savage and } \\
\text { McEneaney. } \\
2020(20)\end{array}$ \\
\hline 55 & $\begin{array}{l}\text { Diltiazem, } \\
\text { Hidralazina, } \\
\text { Bumetanide, }\end{array}$ & 5.4 & 13.5 & $\begin{array}{l}\text { FC: } 30-40 \\
\text { PA: } 82 / 37\end{array}$ & $\begin{array}{l}\text { Gluconato de calcio, } \\
\text { insulina + dextrosa, } \\
\text { nebulziación con }\end{array}$ & $\begin{array}{l}\text { Arif et al. } \\
2020 .(16)\end{array}$ \\
\hline
\end{tabular}




\begin{tabular}{|c|c|c|c|c|c|c|}
\hline & $\begin{array}{l}\text { Metolazone, } \\
\text { atorvastatina, } \\
\text { Glargina }\end{array}$ & & & & $\begin{array}{l}\text { albuterol, sulfonato } \\
\text { de poliestireno } \\
\text { sódico, atropina, } \\
\text { marcapasos } \\
\text { transvenoso, } \\
\text { dopamina, TRR. }\end{array}$ & \\
\hline 60 & $\begin{array}{l}\text { Amlodipina, } \\
\text { atenolol }\end{array}$ & 6.19 & 7.6 & $\begin{array}{l}\text { FC: } 32 \\
\text { PA: } 100 / 70\end{array}$ & $\begin{array}{l}\text { Atropina, gluconato } \\
\text { de calcio, insulina } \\
\text { con dextrosa, } \\
\text { bicarbonato, } \\
\text { nebulización con } \\
\text { salbutamol, } \\
\text { isoproterenol, TRR. }\end{array}$ & $\begin{array}{l}\text { Vishnu et al. } \\
2021 \text { (17) }\end{array}$ \\
\hline 83 & \begin{tabular}{|l} 
Azilsartan, \\
Bisoprolol, \\
ipragliflozina, \\
metformina.
\end{tabular} & 7.8 & 3.26 & $\begin{array}{l}\text { FC: } 24 \\
\text { PA: } 68 / 44\end{array}$ & $\begin{array}{l}\text { Insulina, gluconato } \\
\text { de calcio }\end{array}$ & $\begin{array}{l}\text { Nagamine } \\
\text { 2021. (11) }\end{array}$ \\
\hline ------ & $\begin{array}{l}\text { Aspirina, } \\
\text { Carvedilol, } \\
\text { Verapamilo }\end{array}$ & 6.8 & 3.01 & $\begin{array}{l}\text { FC: } 33 \\
\text { PA: } 80 / 45\end{array}$ & $\begin{array}{l}\text { Atropina, gluconato } \\
\text { de calcio, insulina, } \\
\text { bicarbonato, } \\
\text { glucagón, } \\
\text { ondansetron }\end{array}$ & $\begin{array}{l}\text { Prabhu et al. } \\
2020 \text { (8) }\end{array}$ \\
\hline 66 & $\begin{array}{l}\text { Aspirina, } \\
\text { atorvastatina, } \\
\text { carvedilol, } \\
\text { clopidogrel, } \\
\text { losartan, } \\
\text { Metformina- } \\
\text { sitagliptina }\end{array}$ & 6.2 & 2.22 & $\begin{array}{l}\text { FC: } 35 \\
\text { PA: } 87 / 62\end{array}$ & $\begin{array}{l}\text { Gluconato de calcio, } \\
\text { insulina en dextrosa } \\
\text { IV }\end{array}$ & $\begin{array}{l}\text { Sattar et al. } \\
2020(15)\end{array}$ \\
\hline 74 & $\begin{array}{l}\text { Metoprolol, } \\
\text { Lisinopril. }\end{array}$ & 7.1 & 3.09 & $\begin{array}{l}\text { FC: } 40 \\
\text { PA: } 60 / 30\end{array}$ & $\begin{array}{l}\text { Epinefrina, albuterol, } \\
\text { calcio, insulina iv, } \\
\text { TRR. }\end{array}$ & $\begin{array}{l}\text { Flores } 2020 . \\
(14)\end{array}$ \\
\hline 71 & $\begin{array}{l}\text { Amlodipina, } \\
\text { Nifedipina, } \\
\text { Valsartan, } \\
\text { Carvedilol, } \\
\text { Insulina. }\end{array}$ & 5.8 & 6.23 & $\begin{array}{l}\text { FC: } 31 \\
\text { PA: } 81 / 40\end{array}$ & $\begin{array}{l}\text { Isoproterenol, calcio, } \\
\text { insulina en dextrosa } \\
\text { IV }\end{array}$ & $\begin{array}{l}\text { Park et al. } \\
\text { 2021. (18) }\end{array}$ \\
\hline 51 & $\begin{array}{l}\text { Carvedilol, } \\
\text { eplerenona, } \\
\text { trimetoprima/sulf } \\
\text { ametoxazol }\end{array}$ & 8.6 & 3.3 & $\begin{array}{l}\text { FC: } 20 \\
\text { PA: } 60 / 30\end{array}$ & $\begin{array}{l}\text { Atropina, calcio, } \\
\text { bicarbonato, } \\
\text { albuterol, insulina en } \\
\text { dextrosa IV, } \\
\text { hidrocortisona. }\end{array}$ & $\begin{array}{l}\text { Diribe } 2019 . \\
(13)\end{array}$ \\
\hline
\end{tabular}




\begin{tabular}{|l|l|l|l|l|l|l|}
\hline 62 & $\begin{array}{l}\text { Temisartan, } \\
\text { Atenolol, } \\
\text { Diltiazem, } \\
\text { Furosemida, } \\
\text { Metformina, } \\
\text { Aspirina. }\end{array}$ & 6.3 & 1.9 & $\begin{array}{l}\text { FC: } 40 \\
\text { PA: 105/41 }\end{array}$ & $\begin{array}{l}\text { Gluconato de calcio, } \\
\text { insulina en dextrosa } \\
\text { IV dopamina, } \\
\text { epinefrina }\end{array}$ & $\begin{array}{l}\text { Wong and } \\
\text { Jaafar. 2021 } \\
(10)\end{array}$ \\
\hline $\begin{array}{l}\text { Diltiazem, } \\
\text { Furosemida, } \\
\text { Metoprolol, } \\
\text { Felodipina, } \\
\text { espironolactona, } \\
\text { aspirina, } \\
\text { vidagliptina, } \\
\text { fumarato ferroso }\end{array}$ & 5.5 & 2.9 & $\begin{array}{l}\text { FC:48 } \\
\text { PA:88/52 }\end{array}$ & $\begin{array}{l}\text { Atropina, dopamina, } \\
\text { insulina en dextrosa } \\
\text { IV gluconato de } \\
\text { calcio marcapasos } \\
\text { transcutáneo }\end{array}$ & $\begin{array}{l}\text { Wong and } \\
\text { Jaafar. 2021 } \\
\text { (10) }\end{array}$ \\
\hline
\end{tabular}

Abreviaturas: FC:Frecuencia cardíaca, PA= Presión arterial en mmHg, TRR= terapia de Remplazo Renal.

En un análisis retrospectivo realizado en pacientes admitidos al servicio de emergencias de un hospital suizo durante el 2017 y el 2018, se identificó aquellos pacientes que cumplían criterios para síndrome de BRASH y se observó que la media de niveles de potasio fue de $5.8 \mathrm{mEq} / \mathrm{L}$ (4).

En el otro extremo, el caso de intoxicación con fármacos bloqueadores de la conducción del nodo atrioventricular de forma aislada se diferencia del síndrome de BRASH en la historia clínica; en los primeros hay un historial de una intoxicación con estos fármacos; en el síndrome de BRASH, el paciente, por lo general, se encuentra con un apego terapéutico adecuado, no hay historia de administración supraterapéutica de los medicamentos y, en el caso de este síndrome, el rol de estos medicamentos no es dosis dependiente, indicando nuevamente la naturaleza sinergista del síndrome $(1,5)$.

\section{CLÍNICA}

La presentación clínica de los pacientes es altamente variable, no hay síntomas o signos específicos. Según los reportes de casos, los pacientes pueden ser completamente asintomáticos o presentarse con síntomas que se describen como fatiga generalizada, debilidad, letargo, náuseas, confusión, vértigo, disnea y síncopes $(5,6,8,10-13,19)$. Los puntos clave en la presentación del paciente con síndrome de BRASH es una historia clínica detallada con énfasis en el historial de tratamientos farmacológicos, la bradicardia profunda y la hiperkalemia. Es de notar que el paciente con síndrome de BRASH se presenta de forma usual con una apariencia física que puede ser incongruente con las alteraciones en los signos vitales y las hidroelectrolíticas (1). A razón de lo anterior, se recalca la importancia de tener siempre presente este síndrome dentro del diagnóstico diferencial cuando se va a abordar un paciente con síntomas variados inespecíficos, bradicardia e hiperkalemia. El otro extremo de la presentación es aquel paciente completamente asintomático que tiene como tratamiento de base fármacos con múltiples interacciones farmacológicas quienes desencadenan un síndrome de BRASH secundario a un cuadro infeccioso, como lo fuera una neumonía, o deshidratación por 
una enfermedad diarreica llevando con mínimos cambios electrolíticos a un cuadro clínico severo de bradicardia sintomática, choque y paro cardio respiratorio.

\section{ABORDAJE DIAGNÓSTICO}

El diagnóstico de un síndrome de BRASH requiere de una alta sospecha clínica, especialmente porque son diversas las alteraciones y, si no se contemplan, puede que no se vea el panorama completo cayendo en el letal error de tratar un aspecto del cuadro de manera aislada. Bradicardia, independientemente de la causa, debe confirmarse mediante un electrocardiograma de 12 derivaciones; permite corroborar que se trate de una bradicardia, pero también arroja información acerca del mecanismo desencadenante y la condición eléctrica del corazón del paciente, proporcionando a, su vez, posibles blancos terapéuticos (5).

La utilización del electrocardiograma para constatar hiperkalemia en un posible síndrome de BRASH, o hiperkalemia per se, puede llevar al clínico a formular una impresión diagnóstica falsa $(4,6,10,17)$. Como se ha mencionado previamente, los cambios morfológicos representativos de hiperkalemia en el electrocardiograma, por lo general, no se exhiben, en parte porque los niveles no son lo suficientemente elevados para generar cambios electrocardiográficos; también porque la sensibilidad del EKG como método para detectar hiperkalemia es deficiente $(1,5,9,17)$. Con base en lo anterior, es necesario para el diagnóstico y manejo del síndrome de BRASH la solicitud de un panel metabólico, no solamente evalúa niveles de potasio sérico, también sirve para evaluar la función renal.

La función renal brinda información importante más allá de ser criterio diagnóstico para síndrome de BRASH, permite establecer la función renal basal del paciente, con ello hacer distinción entre una lesión renal aguda y una lesión renal crónica reagudizada. A propósito de la función renal, otro examen de laboratorio por realizar es el de gases arteriales de forma seriada durante el abordaje del paciente. La pérdida de la función renal arrastra consigo la habilidad del cuerpo para excretar aniones y compuestos ácidos causando acidosis metabólica con brecha aniónica elevada e incremento en el lactato $(1,5,10,12)$.

\section{TRATAMIENTO}

A raíz de que esta entidad se presenta como un síndrome, a razón de sus múltiples signos y síntomas, esto puede dirigir al clínico a enfocar sus esfuerzos terapéuticos en la implementación de intervenciones que corrigen un aspecto singular del síndrome, omitiendo el cuadro clínico completo del paciente y la fisiopatología subyacente. Ejemplo de ello es la implementación del algoritmo de manejo de bradicardias de la Advanced Cardiovascular Life Support (ACLS) de la Sociedad Americana del Corazón, el cual no ha ofrecido resultados satisfactorios al utilizarse para tratar la bradicardia del síndrome de BRASH. Lo anterior muestra la importancia de tener una comprensión fisiopatológica del síndrome.

La utilización de atropina en bradicardia supone que el origen proviene de un efecto mediado por el sistema nervioso parasimpático; sin embargo, la fisiopatología del síndrome de BRASH no está relacionada con el aumento del tono parasimpático y al no haber respuesta esperada con la atropina, se continúa con el algoritmo cayendo en la sobreutilización de marcapasos $(1,9)$. Muestra de ello son los casos reportados donde se realizó dicha intervención inicial sin respuesta clínica satisfactoria e incluso la muerte $(8,10,13,16,17,19,20)$. 
El abordaje, por lo tanto, es dirigido a romper el ciclo vicioso tratando de forma simultánea los problemas del paciente. Por este motivo, el manejo consiste en el tratamiento de la hiperkalemia, bradicardia, prevenir el deterioro de la función renal y el choque.

Las estrategias de tratamiento para el manejo de la hiperkalemia constan de medidas estándar.

La estabilización de membrana miocárdica mediante administración de calcio, ya sea mediante uso de gluconato de calcio o, en su defecto, cloruro de calcio mediante una vía central y aquellos que estén en paro cardíaco, a razón de que este último contiene tres veces más cantidad de calcio (5). Este es otro de los aspectos que no contempla el algoritmo de manejo de bradicardia de la ACLS. Medidas de redistribución intracelular de potasio con insulina y dextrosa.

Adicionalmente, considerar nebulización con albuterol, ya que tiene actividad sobre la hiperkalemia y puede mejorar la bradicardia por su efecto secundario $(1,5,9)$. Es usual que estas medidas sean suficientes, sin embargo, en casos refractarios, se ha requerido de terapia de reemplazo renal para mejorar los niveles de hiperkalemia mediante diálisis $(6,9,12,14,16,17)$. Es prudente hacer la observación de que la hiperkalemia siempre debe ser tratada, aunque sea leve. Nuevamente, el mecanismo fisiopatológico es sinergista, no aditivo (1).

El empleo de calcio IV contrarresta los efectos cardiotóxicos que produce la hiperkalemia, mejorando la bradicardia. En casos en los cuales no se produzca el efecto esperado, la opinión de expertos es que no se debe titubear el empleo de epinefrina de forma temprana como dosis push, y en paciente chocado utilizado en infusión. En este punto, se recalcan algunos aspectos; primero, el uso de epinefrina aumenta la frecuencia cardíaca, al ser el gasto cardíaco directamente proporcional a la frecuencia cardíaca, esto presume un aumento en el gasto cardíaco, por tanto, una mejora en la función hemodinámica y la perfusión renal. A su vez, la epinefrina, por su efecto sobre receptor beta 2 del músculo esquelético, causa redistribución intracelular del potasio $(1,2,5,6,9)$. No obstante, en presencia de falla renal, los niveles plasmáticos de renalasa, enzima que metaboliza epinefrina, se verán disminuidos provocando aumento de niveles plasmáticos de epinefrina y con ello la vasoconstricción de arterias renales, culminando en empeoramiento de la isquemia renal (17). Con relación a lo anterior, si dicho efecto perjudicial es significativo o no, al punto de no utilizarse la epinefrina en síndrome de BRASH, necesitará de futura investigación.

Actualmente, no hay protocolos de tratamiento establecidos y la información con la que se cuenta es la de opinión de expertos; sobre este aspecto, dicha opinión recomienda el uso de epinefrina.

En los casos de pacientes donde el uso de epinefrina falló en lograr objetivos terapéuticos, se recomienda el uso de isoproterenol, un fármaco beta-agonista no selectivo. Gracias a su perfil betaadrenérgico puro, tiene mínimo efecto sobre receptores alfa y mayor efecto cronotrópico positivo que otros fármacos, por ejemplo, dobutamina. Se sugiere que la dosis de epinefrina o isoproterenol se titulen para alcanzar una frecuencia cardíaca de 60 a 80 latidos por minuto $(1,5,10,13,17,20)$.

Con respecto al uso de fluidoterapia, cada paciente debe ser estratificado de acuerdo con su estado de volemia. Si bien es cierto, muchos casos de síndrome de BRASH son desencadenados por hipovolemia, por lo tanto, se verán beneficiados de reanimación 
con soluciones IV; otros, sin embargo, entran en una falla renal oligúrica, donde la reanimación generará sobrecarga de volumen. El uso de solución dextrosa al 5\% en agua más bicarbonato de sodio es la solución elegida en pacientes que cursan con acidosis e hiperkalemia. Se ha demostrado que el uso de bicarbonato en acidosis, aunque no disminuye mortalidad, disminuye morbilidad; en este caso particular, se ha visto que su uso circunviene o atrasa la necesidad inmediata de dializar al paciente, y la redistribución equitativa de la solución D5W entre espacios permite, a su vez, redistribución del potasio.

En pacientes que cursan hipovolémicos sin acidosis, se puede utilizar soluciones balanceadas. Cabe señalar aquí que se debe evitar la solución "salina normal", en tanto produce acidosis metabólica, brecha aniónica no elevada provocando movimiento de potasio del intracelular al extracelular empeorando la hiperkalemia(1,5,6,8,9,13). Al alcanzar una volemia adecuada y en aquellos casos donde la severidad de la insuficiencia renal y la hiperkalemia requieran de medidas más avanzadas, se recomienda el empleo de diuréticos con efecto sobre la excreción de potasio, siempre y cuando la hipovolemia haya sido revertida. En caso de que estas medidas fallen en resolver la hiperkalemia, se requerirá de manejo mediante diálisis $(1,6,9,12,14,16-18)$.

\section{CONCLUSIONES}

La importancia del síndrome de BRASH radica en que es una complicación que pone en riesgo la vida de los pacientes; si no se inician medidas para su manejo de forma expedita, la naturaleza cíclica de esta lleva rápidamente al paro cardíaco, y sin embargo, a pesar de su severidad, se requiere de un alto índice de sospecha clínica para lograr identificarlo y corregirlo. A pesar de lo anterior, el síndrome de BRASH, en la actualidad, es una condición pobremente diagnosticada, que puede pasar completamente desapercibida, de modo que no solo se requiere conocimiento del síndrome, el clínico para identificar aquellos pacientes que posean riesgo para desencadenarlo ocupan de un abordaje donde se haga una inspección minuciosa en los tratamientos farmacológicos que utilizan los pacientes para detectar interacciones entre los mismos. Es por ello que con el fin de aumentar el índice de sospecha se presenta en esta revisión los fármacos mayormente involucrados en la génesis del síndrome de BRASH. Tabla 2

Síndrome de BRASH no es una condición que concierne únicamente al personal de servicios de emergencias; se ha descrito su aparición tanto en pacientes sometidos a exámenes en laboratorios de cardiología clínica, así como intraoperatoriamente en sala de operaciones; por esta razón, la importancia de conocer sobre este síndrome y su manejo en las distintas áreas de la praxis médica.

\section{Los autores declaran no tener conflicto de interés.}

\section{REFERENCIAS}

1. Farkas JD, Long B, Koyfman A, Menson K. BRASH Syndrome: Bradycardia, Renal Failure, AV Blockade, Shock, and Hyperkalemia. J Emerg Med. 2020 Aug;59(2):216-23.

2. Schnaubelt $S$, Roeggla M, Spiel AO, Schukro C, Domanovits $\mathrm{H}$. The BRASH syndrome: an interaction of bradycardia, renal failure, AV block, shock and hyperkalemia. Intern Emerg Med. 2021 Mar;16(2):509-11.

3. Halli-Tierney A, Scarbrough C, Carroll DG. Polypharmacy: Evaluating Risks and Deprescribing. Am Fam Physician. 2019 Jul 1;100(1):32-8. 
4. Ravioli S, Woitok BK, Lindner G. BRASH syndrome - fact or fiction? A first analysis of the prevalence and relevance of a newly described syndrome. Eur J Emerg Med. 2021 Apr;28(2):153-5.

5. Lizyness K, Dewald O. BRASH Syndrome. In: StatPearls [Internet]. Treasure Island (FL): StatPearls Publishing; 2021 [cited 2021 Oct 5]. Available from: http://www.ncbi.nlm.nih.gov/books/NBK570643/

6. Sarkar S, Rapista N, Rout A, Chaudhary R. BRASH: Case Report and Review of Literature. Hearts. 2021 Jun 27;2(3):302-6.

7. Srivastava S, Kemnic T, Hildebrandt KR. BRASH syndrome. BMJ Case Rep. 2020 Feb;13(2):e233825.

8. Prabhu V, Hsu E, Lestin S, Soltanianzadeh Y, Hadi S. Bradycardia, Renal Failure, Atrioventricular Nodal Blockade, Shock, and Hyperkalemia (BRASH) Syndrome as a Presentation of Coronavirus Disease 2019. Cureus [Internet]. 2020 Apr 24 [cited 2021 Oct 6]; Available from: https://www.cureus.com/articles/31127bradycardia-renal-failure-atrioventricular-nodalblockade-shock-and-hyperkalemia-brashsyndrome-as-a-presentation-of-coronavirusdisease-2019

9. Grigorov MV, Belur AD, Otero D, Chaudhary S, Grigorov E, Ghafghazi S. The BRASH syndrome, a synergistic arrhythmia phenomenon. Bayl Univ Med Cent Proc. 2020 Oct 1;33(4):668-70.

10. Wong C, Jaafar M. Bradycardia, renal failure, atrioventricular nodal blockade, shock, and hyperkalemia: An important syndrome to recognize. Turk J Emerg Med. 2021;21(2):86.

11. Nagamine T. BRASH syndrome associated with angiotensin receptor blocker and SGLT2 inhibitor. Can J Emerg Med [Internet]. 2021 Oct 1 [cited 2021 Oct 6]; Available from: https://link.springer.com/10.1007/s43678-02100213-9

12. Ata $F$, yasir $M$, Javed $S$, Bilal ABI, Muthanna B, Minhas $B$, et al. Diagnostic and therapeutic challenges of BRASH syndrome: A case report. Med Case Rep Study Protoc. 2021 Jan;2(1):e0018.

13. Diribe N, Le J. Trimethoprim/SulfamethoxazoleInduced Bradycardia, Renal Failure, AV-Node Blockers, Shock and Hyperkalemia Syndrome. Clin Pract Cases Emerg Med. 2019 Jul 22;3(3):282-5.

14. Flores S. Anaphylaxis induced bradycardia, renal failure, AV-nodal blockade, shock, and hyperkalemia: A-BRASH in the emergency department. Am J Emerg Med. 2020 Sep;38(9):1987.e1-1987.e3.

15. Sattar $Y$, Bareeqa SB, Rauf H, Ullah W, Alraies MC. Bradycardia, Renal Failure, Atrioventricularnodal Blocker, Shock, and Hyperkalemia Syndrome Diagnosis and Literature Review. Cureus [Internet]. 2020 Feb 13 [cited 2021 Oct 6]; Available from:

https://www.cureus.com/articles/27877bradycardia-renal-failure-atrioventricular-nodalblocker-shock-and-hyperkalemia-syndromediagnosis-and-literature-review

16. Arif AW, Khan MS, Masri A, Mba B, Ayub MT, Doukky R. BRASH Syndrome with Hyperkalemia: An Under-Recognized Clinical Condition. Methodist DeBakey Cardiovasc J. 2020 Jul 1;16(3):241.

17. Vishnu V, Jamshed N, Amrithanand V, Thandar S. BRASH Syndrome: A Case Report. J Emerg Med. 2021 Jun;60(6):818-22.

18. Park JI, Jung MS, Lee $\mathrm{H}$, Kim H, Oh J. Implication of $\mathrm{AV}$ node blockers in patients with end-stage renal disease undergoing head and neck surgery; BRASH syndrome: a case report. Braz J Anesthesiol Engl Ed. 2021 May;S0104001421001998.

19. Barreras N. Brash Syndrome, Can You See the Cycle? In: C44 CRITICAL CARE CASE REPORTS: METABOLIC, RENAL, AND ENDOCRINE [Internet]. American Thoracic Society; 2020 [cited 2021 Oct 4]. p. A5184-A5184. Available from: https://www.atsjournals.org/doi/10.1164/ajrccmconference.2020.201.1 MeetingAbstracts.A5184

20. Savage $P$, McEneaney D. BRASH SYNDROME: AN UNDER RECOGNISED CAUSE OF COMPLETE HEART BLOCK IN THE ELDERLY. Ulster Med J. 2020 Sep;89(2):123-4. 